\title{
Whole-genome resequencing of Osmanthus fragrans provides insights into flower color evolution
}

\author{
Hongguo Chen ${ }^{1,2}$, Xiangling Zeng ${ }^{1,2,3}$, Jie Yang ${ }^{1,2}$, Xuan Cai ${ }^{1,2}$, Yumin Shi ${ }^{1,2}$, Riru Zheng ${ }^{3}$, Zhenqi Wang ${ }^{4}$, Junyi Liu ${ }^{5}$,
} Xinxin $\mathrm{Yi}^{6}$, Siwei $\mathrm{XiaO}^{6}$, Qiang $\mathrm{Fu}^{3}$, Jingjing Zou ${ }^{1,2}$ and Caiyun Wang ${ }^{3}$

\begin{abstract}
Osmanthus fragrans is a well-known ornamental plant that has been domesticated in China for 2500 years. More than 160 cultivars have been found during this long period of domestication, and they have subsequently been divided into four cultivar groups, including the Yingui, Jingui, Dangui, and Sijigui groups. These groups provide a set of materials to study genetic evolution and variability. Here, we constructed a reference genome of $O$. fragrans 'Liuyejingui' in the Jingui group and investigated its floral color traits and domestication history by resequencing a total of 122 samples, including 119 O. fragrans accessions and three other Osmanthus species, at an average sequencing depth of 15x. The population structure analysis showed that these 119 accessions formed an apparent regional cluster. The results of linkage disequilibrium (LD) decay analysis suggested that varieties with orange/red flower color in the Dangui group had undergone more artificial directional selection; these varieties had the highest LD values among the four groups, followed by the Sijigui, Jingui, and Yingui groups. Through a genome-wide association study, we further identified significant quantitative trait loci and genomic regions containing several genes, such as ethylene-responsive transcription factor 2 and Arabidopsis pseudoresponse regulator 2, that are positively associated with petal color. Moreover, we found a frameshift mutation with a 34-bp deletion in the first coding region of the carotenoid cleavage dioxygenase $4 \mathrm{gene}$. This frameshift mutation existed in at least one site on both alleles in all varieties of the Dangui group. The results from this study shed light on the genetic basis of domestication in woody plants, such as $O$. fragrans.
\end{abstract}

\section{Introduction}

Sweet osmanthus (Osmanthus fragrans Lour.), belonging to the family Oleaceae, is a well-known ornamental germplasm native to the Sino-Himalayan region ${ }^{1}$. It has been cultivated in China for more than 2500 years. More than 160 cultivars of $O$. fragrans have been classified based on phenotypes, such as flower color and blooming

Correspondence: Jingjing Zou (silence@hbust.edu.cn) or

Caiyun Wang (wangcy@mail.hzau.edu.cn)

${ }^{1}$ Hubei Engineering Research Center for Fragrant Plants, Hubei University of

Science and Technology, Xianning 437100, China

${ }^{2}$ Xianning Research Academy of Industrial Technology of Osmanthus fragrans, Xianning 437100, China

Full list of author information is available at the end of the article

These authors contributed equally: Hongguo Chen, Xiangling Zeng season. They have been divided into four cultivar groups, including the Yingui group (Albus group), which has white to pale yellow flowers; the Jingui group (Luteus group), which has yellow flowers; the Dangui group (Aurantiacus group), which has orange/red flowers that bloom mainly in autumn for commercial harvest; and the Sijigui group (Asiaticus group), which has pale yellow to yellow flowers that bloom throughout most of the year $^{2-4}$. It is thought that varieties in the Sijigui group and Yingui group are less differentiated from wild $O$. fragrans than the other two groups, which probably originated earlier ${ }^{2}$. The results of microsatellite marker analysis indicate that the varieties in the Jingui and Dangui groups, which displayed more significant genetic

\section{(c) The Author(s) 2021}

(c) (i) Open Access This article is licensed under a Creative Commons Attribution 4.0 International License, which permits use, sharing, adaptation, distribution and reproduction cc) in any medium or format, as long as you give appropriate credit to the original author(s) and the source, provide a link to the Creative Commons license, and indicate if changes were made. The images or other third party material in this article are included in the article's Creative Commons license, unless indicated otherwise in a credit line to the material. If material is not included in the article's Creative Commons license and your intended use is not permitted by statutory regulation or exceeds the permitted use, you will need to obtain permission directly from the copyright holder. To view a copy of this license, visit http://creativecommons.org/licenses/by/4.0/. 
differentiation, might have diverged earlier ${ }^{4}$. Thus, the evolutionary relationships of varieties with different colors are still not clear.

Due to their ornamental and commercial value, flowers have long been a focus of interest in the study of $O$. fragrans. It has been reported that $\alpha$-ionone and $\beta$-ionone are the main floral components of $O$. fragrans ${ }^{5-7}$. The accumulation of $\alpha$-ionone and $\beta$-ionone in the cultivars of the Yingui, Jingui, and Sijigui groups is higher than that in the Dangui group, mainly due to the higher efficiency of carotenoid cleavage ${ }^{8,9}$. Furthermore, the presence of white, yellow, and orange color varieties is primarily attributable to the level of carotenoids, whereas flavonoids are speculated to provide only the background color $^{10}$. Thus, the main differences in flower color and floral fragrance among varieties in different groups of $O$. fragrans are determined mainly by the degree of carotenoid accumulation and cleavage. Carotenoid cleavage dioxygenase $1(C C D 1)$ and $C C D 4$ are crucial contributors to the cleavage of $\alpha$-carotene and $\beta$-carotene into $\alpha$-ionone and $\beta$-ionone ${ }^{9,11}$. The most critical factor determining the diversity of carotenoid concentrations was the differential expression level of $C C D 4^{10,12}$. This leads to the question, what role does the $C C D 4$ gene play in the evolution of $O$. fragrans flower color?

More recently, genome sequencing of $O$. fragrans 'Rixianggui' (OFR) in the Sijigui group, which blooms for most of the year, has been performed at the chromosome level ${ }^{13}$. However, a systematic study to chart the genetic architecture of ornamental traits in a large population using a genome-wide association (GWA) method has not yet been performed. As most cultivars of $O$. fragrans bloom in autumn, we generated a reference genome for $O$. fragrans 'Liuyejingui' (OFL) from the Jingui group. In addition to the flowering time, OFR has fewer flowers at each blooming event, with a typical complete pedicel and pale yellow flower color. OFL produces many flowers that typically bloom for a week twice per year on average; the flowers are lemon yellow in color, with a strong fragrance and high essential oil contents, and are harvested for ornamental use as well as food and industrial uses $^{14,15}$. We also reported on genomic variations and population evolution by resequencing 119 O. fragrans accessions with different colors from the four groups. We further sequenced the transcriptomes of different tissues of OFL, such as the rhizomes, leaves, flowers, and flowers, in different flowering stages to validate the quantitative trait loci (QTLs) and functional CCD4s through the expression of candidate genes between transcriptomes. For the first time, the present study explains the origin and evolutionary relationship of varieties in different groups of $O$. fragrans and color formation in the different varieties in terms of the deletion of the $C C D 4$ gene structure.

\section{Materials and methods \\ Plant materials}

For genome sequencing, leaf samples were collected from OFL on the campus of Huazhong Agricultural University (Wuhan, China) $\left(114^{\circ} 21^{\prime} \mathrm{W}, 30^{\circ} 29^{\prime} \mathrm{N}\right)$. For resequencing, leaves were collected from 119 representative $O$. fragrans landraces and three close relatives of osmanthus, including O. cooperi, $O . \times$ fortunei, and $O$. heterophyllus (G. Don) P. S. Green var. Heterophyllus (Supplementary Table 1).

\section{Genome sequencing and resequencing}

Fresh, healthy leaves were harvested from the bestgrowing individuals and immediately frozen in liquid nitrogen, followed by preservation at $-80^{\circ} \mathrm{C}$ in the laboratory prior to DNA extraction. High-quality genomic DNA was extracted using a modified Cetyltrimethyl Ammonium Bromide method ${ }^{16}$. For genome sequencing, single-molecule real-time (SMRT) libraries were constructed and sequenced using a PacBio Sequel II instrument (Pacific Biosciences, Menlo Park, CA, USA) at Frasergen Bioinformatics Co., Ltd. (Wuhan, China). For resequencing, $1 \mu \mathrm{g}$ DNA per sample was used as the input material, and sequencing libraries were generated using the VAHTS Universal DNA Library Prep Kit for MGI (Vazyme, Nanjing, China) following the manufacturer's recommendations. Library quantification and size measurement were performed using a Qubit 3.0 Fluorometer (Life Technologies, Carlsbad, CA, USA) and a Bioanalyzer 2100 system (Agilent Technologies, CA, USA). Subsequently, libraries of 122 accessions were constructed and sequenced on an MGI-SEQ 2000 platform at Frasergen Bioinformatics Co., Ltd.

\section{Transcriptome sequencing}

To obtain information that assists in the annotation of genes, the Iso-Seq method was performed to produce fulllength transcripts using SMRT sequencing ${ }^{17}$. RNA was prepared from flowers, leaves, stems, and roots collected from the same tree and processed for library construction. Total RNA was extracted using TRIzol reagent (Invitrogen) according to the manufacturer's protocol. RNA-seq libraries were prepared using the Clontech SMARTer cDNA synthesis kit according to the manufacturer's recommendations and were then sequenced on the MGISEQ 2000 platform at Frasergen Bioinformatics Co., Ltd. and Igenebook Bioinformatics Institute (Wuhan, China).

\section{Genome assembly}

PacBio SMRT sequencing technology and a highthroughput chromatin conformation capture (Hi-C)-based scaffolding method were used to perform chromosomelevel assembly of the OFL genome. With one SMRT cell in the PacBio Sequel platform, we generated $174.53 \mathrm{~Gb}$ 
subreads by removing adaptor sequences within sequences. The longest 150X subread data were used for the genome assembly of $O$. fragrans. The initial assembly results were generated by using the default parameters of the mecat2 tool with the longest 150X subread data. To correct errors in the primary assembly, we used the Racon (v1.3.1) ${ }^{18}$ pipeline to refine the genome. Finally, we used Illuminaderived short reads to correct any remaining errors by Pilon $(\mathrm{v} 1.22)^{19}$. The short reads from the Illumina platform were quality filtered by HTQC (v1.92.310) ${ }^{20}$.

For anchored contigs, clean read pairs generated from the Hi-C library were mapped to the polished OFL genome using BWA (bwa-0.7.17). Paired reads with mates mapped to a different contig were used to perform Hi-Cassociated scaffolding. Contigs were then successfully clustered into 23 groups with the agglomerative hierarchical clustering method in Lachesis ${ }^{21}$. Lachesis was further applied to order and orient the clustered contigs.

\section{Annotation of repetitive sequences}

Two methods were combined to identify the repeat contents in our genome: homology-based analysis and de novo prediction. With homology-based analysis, we identified the known transposable elements (TEs) within the OFL genome using RepeatMasker (open-4.0.9) ${ }^{22}$ with the Repbase TE library ${ }^{23}$. RepeatProteinMask searches were also conducted using the TE protein database as a query library. By de novo prediction, we constructed a de novo repeat library of the OFL genome using RepeatModeler, which automatically executed two core de novo repeat-finding programs, RECON $(\mathrm{v} 1.08)^{24}$ and RepeatScout (v1.0.5) ${ }^{25}$. Furthermore, we performed a de novo search for long terminal repeat (LTR) retrotransposons against the OFL genome sequences using LTR_FINDER $(\mathrm{v} 1.0 .7)^{26}$. We also identified tandem repeats using the Tandem Repeat Finder package ${ }^{27}$ and noninterspersed repeat sequences, including lowcomplexity repeats, satellites and simple repeats, using RepeatMasker. Finally, we merged the library files of the two methods and used RepeatMaker to identify the repeat contents.

\section{Annotation of protein-coding gene}

We predicted the OFL genome's protein-coding genes using three methods: ab initio, homology-based and RNAseq predictions. We used Augustus (v3.3.1) ${ }^{28}$ and Glimmer $^{29}$ to perform ab initio gene prediction. Exonerate (v2.2.0, -model protein2genome-showtargetgff 1$)^{30}$ GeneWise (2.4.1, -trev -genesf -gff -sum) ${ }^{31}$, and Solar (0.9.6, a prot2genome2 - $\mathrm{n} 200000-\mathrm{z}-\mathrm{f} \mathrm{m} 8)^{32}$ were used to conduct homology-based gene prediction. To carry out RNA-seq-aided gene prediction, we first assembled clean RNA-seq reads into transcripts using TopHat $(\mathrm{v} 2.1 .1)^{33}$, and the gene structure was formed using Cufflinks (v2.2.1,
-I 300000 -p 4 -L CUFF4) ${ }^{34}$. To obtain a more complete gene structure, we also used Iso-seq data. First, the sequencing data were made redundant by CD-HIT (v4.6.7, -AL 1000 -AS 100 -G 0 -M 2500 -aL 0.85 -aS 0.98 -c $0.98-\mathrm{T} 15)^{35}$. Then, the reference genome was compared with GMAP (v2018-07-04, -n 5 -min-intronlength $=9$--max-intronlength-middle $=200000$-t 15 -A -f 2) ${ }^{36}$. Finally, TransDecoder (v5.3.0, default) (http:// transdecoder.sourceforge.net/) structure prediction was performed. Finally, Maker (v3.00) ${ }^{37}$ was used to integrate the three methods' prediction results to predict the genes.

Gene functions were inferred according to the best match of the alignments to the National Center for Biotechnology Information (NCBI) non-redundant, TrEMBL $^{38}$, InterPro ${ }^{39}$. Swiss-Prot ${ }^{38}$, and Kyoto Encyclopedia of Genes and Genomes (KEGG) databases ${ }^{40}$ using BLASTP (NCBI BLAST v2.6.0+ $)^{41,42}$ with an $e$ value threshold of $1 \mathrm{E}^{-5}$. The protein domains were annotated using PfamScan (pfamscan_version) ${ }^{43}$ and InterProScan $(\mathrm{v} 5.35-74.0)^{44}$ based on InterPro protein databases. The motifs and domains within gene models were identified using PFAM databases ${ }^{45}$. Gene Ontology (GO) ${ }^{46}$ IDs for each gene were obtained from Blast $2 \mathrm{GO}^{47}$.

\section{Annotation of noncoding RNA genes}

We used transfer RNA (tRNA)scan-SE (v1.3.1) ${ }^{48}$ algorithms with default parameters to identify the genes associated with tRNA. For ribosomal RNA (rRNA) identification, we first downloaded rRNA sequences from closely related species from the Ensembl database. Then, rRNAs in the database were aligned against our genome using BLASTN ${ }^{41,42}$ with a cutoff of $e$ value $<1 \mathrm{e}^{-5}$, identity $\geq 85 \%$ and match length $\geq 50$ bp. microRNA (MiRNAs) and small nuclear RNAs (snRNAs) were identified by Infernal (v1.1.2) ${ }^{49}$ software against the Rfam (v14.1) ${ }^{45}$ database with default parameters.

\section{Gene family identification}

All proteins were extracted and aligned to each other using BLASTP programs (ncbi blast v2.6.0) ${ }^{42}$ with a maximum $e$ value of $1 \mathrm{e}^{-5}$. To exclude putative fragmented genes, identities with less than $30 \%$, coverage less than $50 \%$, and genes encoding protein sequences that were shorter than $50 \mathrm{bp}$ were filtered out. The OrthoMCL $(\mathrm{v} 14-137)^{50}$ method was used to cluster genes from these different species into gene families with the parameter "-inflation 1.5."

\section{Phylogenetic and gene family analysis}

The single-copy orthologous gene protein sequences were aligned with the MUSCLE (v3.8.31) ${ }^{51}$ program, and the corresponding coding DNA sequence alignments were generated and concatenated with the guidance of the protein alignment. RAxML $(\mathrm{v} 8.2 .11)^{52}$ was used to 
construct the phylogenetic tree with the maximum likelihood method.

Based on the identified gene families and the constructed phylogenetic tree with a predicted divergence time of those species, we used $\mathrm{CAFE}^{53}$ to analyze gene family expansion and contraction. This method implemented hypergeometric test algorithms, and the $q$ value (false discovery rate (FDR)) was calculated to adjust the $p$ value using the $R$ package.

\section{Synteny analyses}

We first performed a whole-genome comparison of the two genomes with the default parameters in the NUCmer tool $^{54}$, filtered the sequence by delta filtering with the -1 parameter, and filtered out collinear fragments with a length less than $10 \mathrm{~kb}$. SNPs and the variations between the two genomes were found using show-snps with the "-rT" parameter and show-diff with the "-rH" parameter, respectively.

\section{Genomic variations}

To explore genetic variations in the $O$. fragrans germplasm, clean reads from the resequencing data of the 122 Osmanthus plant accessions were aligned against the OFL genome assembly using Burrows-Wheeler aligner v0.7.17 $(B W A)^{55}$ with default parameters. The 122 accessions were categorized into five groups: the 119 O. fragrans accessions formed the 'Yingui group', 'Jingui group', 'Dangui group', and 'Sijigui group', and an 'outgroup' was formed that included three other Osmanthus accessions together with data for Olea europaea (Supplementary Table 1).

SNP calling was performed using the Genome Analysis Toolkit v4.1.4.1 $1^{56,57}$. Briefly, duplicated reads were annotated using MarkDuplicates under default settings. SNPs and indels for each sample were first called using HaplotypeCaller, setting the ploidy to 2 and ERC to GVCF mode. GVCFs were combined using CombineGVCF with the default settings. The final genotyping of the population was performed using GenotypGVCFs under default settings. The SNPs were filtered for quality to apply the following criteria: quality/depth $<2.0$ || FS $>60.0$ || MQ (quality of the mapped reads of one site) $<40.0$ ।| MQRankSum $<-12.5$ || ReadPosRankSum $<-8.0$. The SNPs in the joint genotyping were further filtered to remove SNP sites with MAF $<0.05$, sequencing depth $<4$, and those that had samples with missing data.

We used Treebest software (v1.9.2) (http://treesoft. sourceforge.net/treebest.shtml) to build an neighborjoining (NJ) phylogenetic tree with a bootstrap of 100 and visualized the tree using $\mathrm{iTOL}^{58}$. GCTA software (v1.91.4 beta3) was used to perform principal component analysis (PCA) with default settings ${ }^{59}$. We also investigated the population structure using ADMIXTURE (v1.3.0), specifying $K$ values ranging from two to eight ${ }^{60}$. The most suitable number of ancestral populations was determined by the $K$ value with the lowest crossvalidation error (CV). PopLDdecay (v3.30) with MaxDist set at 100 was used to calculate the linkage disequilibrium (LD) value of each group ${ }^{61}$.

Population differentiation indices (Fst: Fixation index) between a pair of subpopulations were calculated using VCFtools (v0.1.13) $)^{62}$, with a slide window size of $100 \mathrm{~kb}$ and step size of $10 \mathrm{~kb}$. To identify regions with differentiation, we took the top $5 \%$ regions as candidate regions, from which we performed GO and KEGG enrichment analysis of the genes in these candidate regions.

\section{Genome-wide association study}

GWAS was performed using GAPIT software (v3.0) ${ }^{63}$ based on an mixed linear model and ten principal components as covariates. The SNP association $p$ value was adjusted for the FDR using the Benjamini and Hochberg $\operatorname{method}^{64}$. The $p$ value $-\log _{10(\mathrm{p})} \geq 7$ was used as a significance threshold. The candidate region was selected as $10 \mathrm{~kb}$ upstream and downstream from a significantly associated SNP. Overlapping candidate regions were merged. The results of the GWAS are presented in the form of Manhattan and Q-Q plots. Genes in candidate regions were analyzed based on GO and KEGG enrichment.

\section{Expression analysis of candidate genes}

A qRT-PCR Applied Biosystems 7500 sequence detection system (ABI7500; Thermo Fisher Scientific, Inc.) was used to analyze samples from different tissue parts (root, stem, and leaf) and flowering stages (S1-S6: Bud stage, initial flowering stage, early full flowering stage, full flowering stage, late full flowering stage, abscission stage) of OFL (Supplementary Fig. 1). The qRT-PCR primers were designed using Prime Premier 5 (Supplementary Table 2). The qRT-PCR solution was composed of $2 \mu \mathrm{L}$ of cDNA, $0.8 \mu \mathrm{L}$ of each forward and reverse primer, $10 \mu \mathrm{L}$ of SYBR Mix and 6.4 $\mu \mathrm{L}$ of double-distilled water in a total volume of $15 \mu \mathrm{L}$. Actin's expression level was used as a reference, and qRT-PCR amplification was performed using the following conditions: $94^{\circ} \mathrm{C}$ for $30 \mathrm{~s}$ and 40 cycles of $94^{\circ} \mathrm{C}$ for $10 \mathrm{~s}$ and $60^{\circ} \mathrm{C}$ for $30 \mathrm{~s}$. Relative expression levels were calculated using the $2^{-\Delta \Delta C T}$ method, and each analysis included three replicates. Significant differences were obtained using SPSS with Duncan's test at $p<0.05$.

\section{Results \\ Genome sequencing and assembly}

The genome size was estimated by flow cytometry using the method of Dolezel ${ }^{65}$ on a Sysmex CyFlow Ploidy Analyzer (Sysmex Medical Electronics Shanghai Co., 
Ltd.). The results suggested that the genome size of OFL was $\sim 690 \mathrm{M}$ by referencing Solanum lycopersicum and $770 \mathrm{M}$ by referencing $S$. tuberosum (Supplementary Fig. 2). OFL was sequenced to obtain $71 \mathrm{~Gb}$ of clean sequence data using the Illumina platform and $174.53 \mathrm{~Gb}$ using the PacBio sequencing platform. We generated the 17-mer occurrence distribution using the Illumina data and estimated the genome size to be $\sim 783.63 \mathrm{Mb}$. The proportion of repeat sequences and the genome's heterozygosity rate were determined to be $\sim 54.37 \%$ and $1.17 \%$, respectively. A $733 \mathrm{Mb}$ genome was assembled using PacBio data containing 575 contigs with a contig N50 of $2.36 \mathrm{Mb}$, which accounted for $93.54 \%$ of the genome size. The contigs were anchored to 23 pseudochromosomes using $\mathrm{Hi}-\mathrm{C}$ libraries with lengths from $21.89 \mathrm{Mb}$ to $47.60 \mathrm{Mb}$ that anchored $92.41 \%$ of the assembled sequences. The final corrected chromosome-level genome was $677 \mathrm{Mb}$ in size, with 541 contigs. The assembled genome was highly complete, with $96.7 \%$ of Benchmarking sets of Universal Single Copy Orthologs (BUSCOs) (Table 1). To examine assembly integrity, the continuous long read subreads were realigned onto the final assembly using minimap2 (v2.5) with the default parameters. A total of $99.52 \%$ of raw reads could be mapped. Overall, $96.7 \%$ complete and $1.0 \%$ partial of BUSCOs were identified in the assembled genome, indicating a high completion level. To evaluate the accuracy of the genome at the nucleotide level, Illumina short reads were aligned to the assembly, and we identified $0.0032 \%$ homozygous SNPs, indicating a highly accurate genome.

Table 1 Comparison of genome sequencing, assembly and annotations of $O$. fragrans 'Liuyejingui' (OFL), and the published $O$. fragrans 'Rixianggui' (OFR)

\begin{tabular}{lll}
\hline Content & OFL genome & OFR genome \\
\hline Genome size & $733.26 \mathrm{Mb}$ & $740.71 \mathrm{Mb}$ \\
Contig number & 575 & 774 \\
Contig N50 & $2.36 \mathrm{Mb}$ & $1.6 \mathrm{Mb}$ \\
Number of superscaffold chromosomes & 23 & 23 \\
Assembled superscaffold & $677.64 \mathrm{Mb}$ & $739.37 \mathrm{Mb}$ \\
chromosomes size & & \\
Asembled superscaffold chromosomes & 541 & - \\
contigs & & \\
Assembled BUSCOs & $96.70 \%$ & $96.10 \%$ \\
Heterozygosity & $1.17 \%$ & $1.45 \%$ \\
Number of genes & 41,252 & 45,542 \\
Average gene length & $5639.29 \mathrm{bp}$ & $4065.24 \mathrm{bp}$ \\
BUSCOs in annotation & $96.80 \%$ & $94.50 \%$ \\
\hline
\end{tabular}

\section{Genome annotations}

We used homology-based and de novo approaches to identify TEs. Our assembly indicated that $447.7 \mathrm{Mb}$ $(61.06 \%)$ of the assembled genome consisted of repeated regions. Among them, LTR retrotransposons were the most abundant annotations, making up $47.78 \%$ of the genome.

We masked repeated regions and proceeded to annotate the genome using a comprehensive strategy including ab initio gene prediction, homology-based gene prediction, and RNA-seq-aided gene prediction. In total, 41,252 protein-coding genes with an average length of $5639 \mathrm{bp}$ were predicted in the assembled OFL genome (Table 1). Approximately 39,068 ( 94.71\%) of the predicted proteincoding genes of OFL were functionally annotated with known genes, conserved domains, and GO terms. In addition, we identified 148 miRNA, 714 tRNA, 500 rRNA, and 248 snRNA sequences.

We used BUSCO to evaluate the quality of our gene annotation and found that 1562 (96.8\%) highly conserved core proteins in embryophyta_odb10 were present in our gene annotation.

\section{Comparative genomics}

To investigate the evolution of OFL, we compared its genome to those of other flowering plant species, including Oryza sativa, Papaver somniferum, Citrus sinensis, Arabidopsis thaliana, Theobroma cacao, Rosa chinensis, Medicago truncatula, Vitis vinifera, Camellia sinensis, Artemisia annua, O. europaea, and Sesamum indicum. As a result, we clustered 41,252 genes into 16,107 gene families. A total of 191 genes were identified as shared single-copy orthologous genes (Fig. 1).

To reveal the phylogenetic relationships among OFL and other related species, protein sequences from the 186 filtered single-copy orthologous genes were used for phylogenetic tree reconstruction. The phylogenetic relationship of the other related species was consistent with that in the previous studies ${ }^{13}$. According to the divergence times and phylogenetic relationships, 4325 gene families were significantly expanded in the OFL genome, and 1851 gene families were significantly contracted $(p<0.05)$. Those expanded gene families included 3274 significantly enriched ( $q$ value $<0.05$ ) KEGG pathways (Fig. 2). Genes involved in the biosynthesis of monoterpenoids, diterpenoids, sesquiterpenoids, triterpenoids, limonene, and carotenoids were expanded.

\section{Genomic variations and population evolution}

The evolutionary relationships of varieties of $O$. fragrans with different flower colors are still unclear. Thus, we collected 119 phenotypically diverse populations of $O$. fragrans cultivars and their close relatives for whole-genome resequencing to investigate the genetic architecture of floral color traits using the OFL genome as a reference genome. 


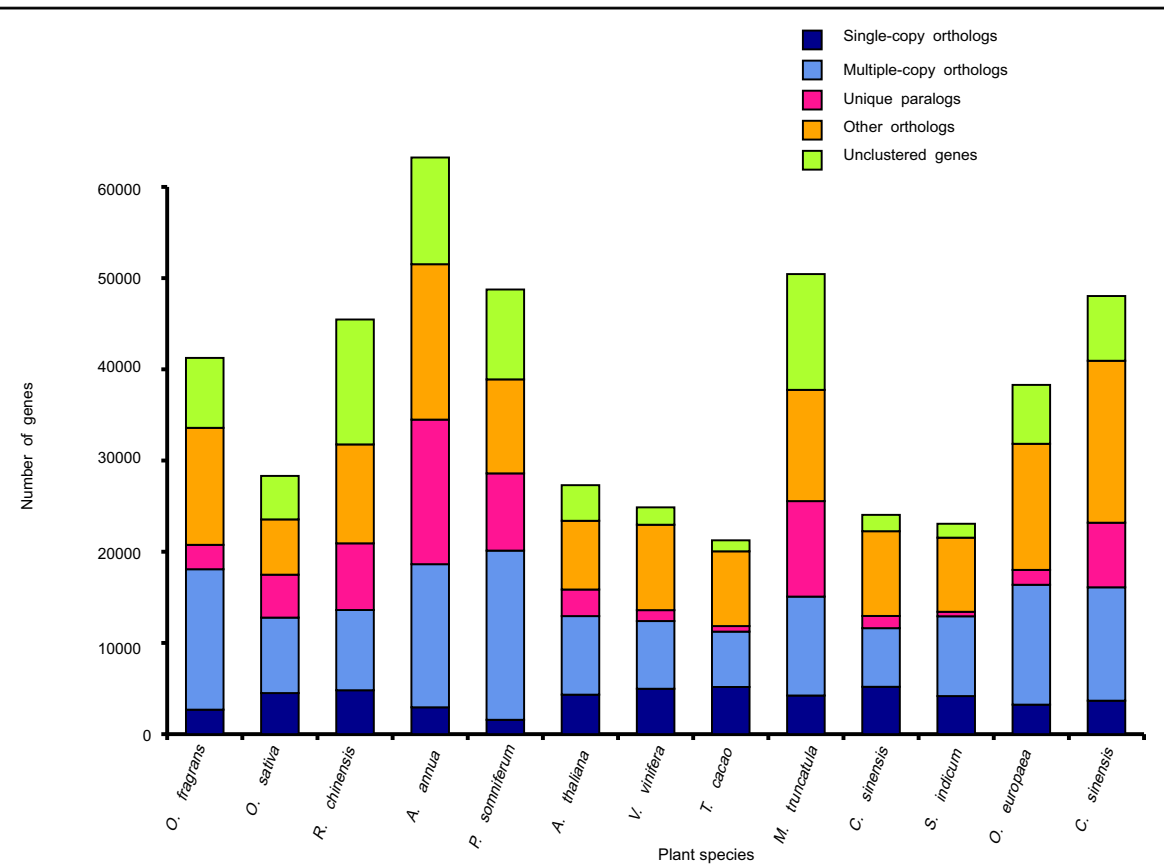

Fig. 1 Evolution of gene numbers. The number of genes in various plant species

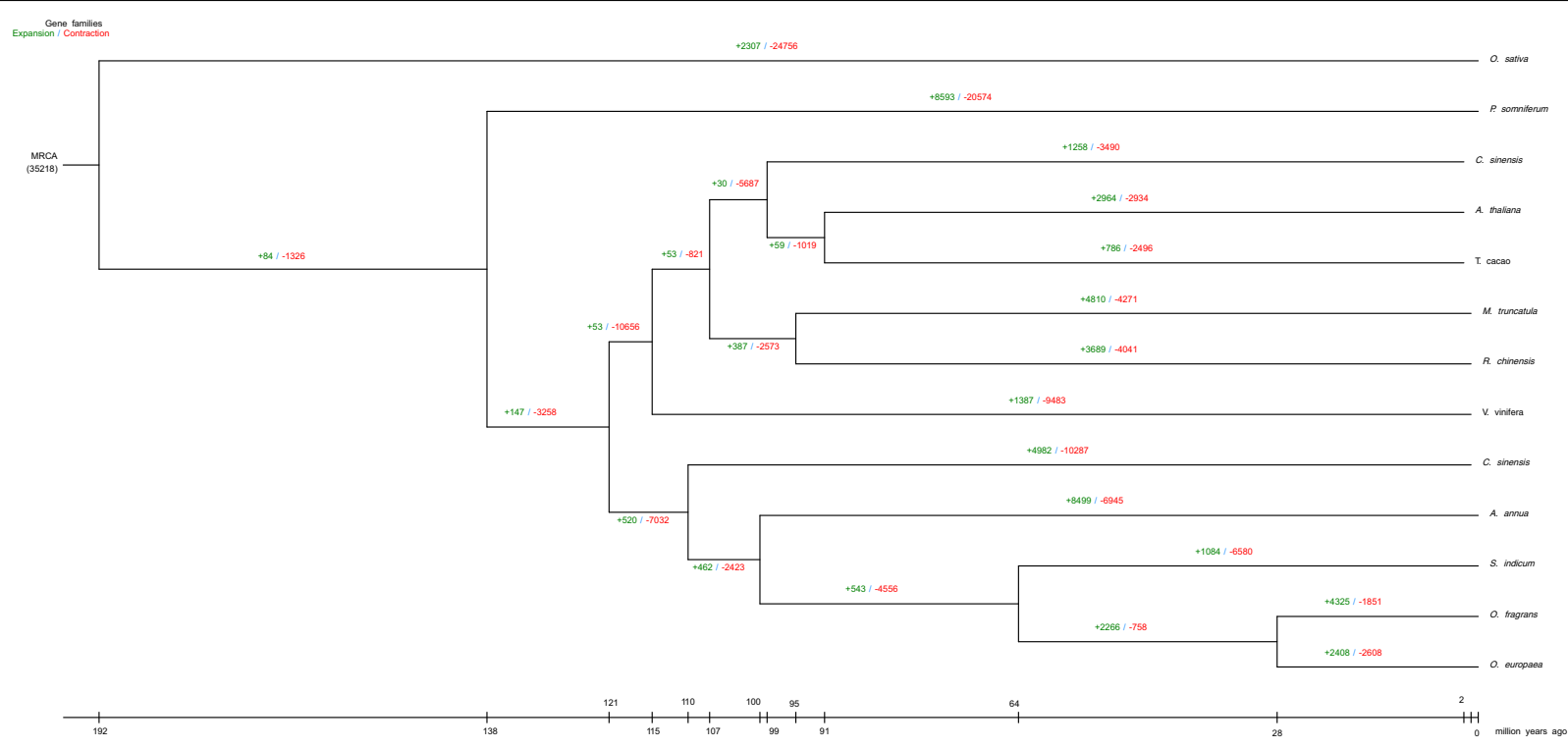

Fig. 2 Gene family expansion and contraction analysis of most recent common ancestor in various plant species. The green number represents the number of expanding gene families and the red number represents the number of contracting gene families

Each plant was subjected to whole-genome sequencing on the MGI-SEQ 2000 platform, obtaining on average $11 \mathrm{G}$ of data per plant, which is approximately $15 \mathrm{X}$ coverage based on the genome size estimates. We mapped the sequencing data to the OFL genome with, on average, a 98\% mapping rate. Approximately $86.7 \%$ of the genome was covered by at least four reads, and $68.6 \%$ of the genome was covered by at least ten reads. We performed SNP calling based on the mapping data and identified 11.44 million SNPs per plant on average. After filtering SNP positions with sequencing depth $<4, \mathrm{MAF}<0.05$ and a requirement of no missing data, we obtained a total of 2,072,100 SNPs.

We constructed a phylogenetic tree using the NJ method. We found three general clusters: cluster A, which 
consisted mainly of the 'Dangui group', and clusters B and $\mathrm{C}$, which consisted predominately of separate subgroups from the 'Yingui group' and 'Sijigui group'. The 'Jingui group' was found to be more dispersed among each of these three tree clusters. From our PCA, we observed similar outcomes, with the 'Dangui group' plants being more separated from other Osmanthus varieties. The 'Yingui group' was separated into two subgroups, both of which clustered more closely with the 'Sijigui group' (Fig. 3a).

The population structure analysis for these populations showed that when the cluster $(\mathrm{k})$ was 8 , the least $\mathrm{CV}$ error was detected. The "Dangui group" showed the lowest amount of mixture, while the other varieties showed some common ancestry from different Osmanthus ancestors (Fig. 3b). The LD decay plots for the four populations showed that the "Dangui group" flattened out the fastest, followed by the "Sijigui group", the "Jingui group", and finally the "Yingui group" (Fig. 3c).

\section{GWAS analysis of ornamental traits}

We examined the important ornamental traits in Osmanthus varieties to look for markers that are significantly associated with petal color.

Osmanthus flower colors are categorized as white, yellow, and orange/red. A total of 22 plants were categorized as having orange/red flowers. The CMLM model identified 25 significant loci containing 35 genes (Fig. 4). The significant candidate regions were distributed on six chromosomes. The identified candidate genes included cytochrome c oxidase (LYG001209), protein transport protein sec16 (SEC16B, LYG008575), ethylene-responsive transcription factor 2 (ERF2, LYG012560), cyclindependent kinase D-1 (LYG012568), auxin response factor 11 (ARF11, LYG014851), E3 ubiquitin-protein ligase (Mib, LYG032877), and 9-cis-epoxycarotenoid dioxygenase (NCED6, LYG034219).

To further filter genes that may contribute to orange/ red flower color in O. fragrans we examined the Fst values between the 'Dangui group' and the other three groups. Of the 35 genes, 24 genes were within the top 5\% of Fst values between the 'Dangui group' and the other groups; these genes included ERF2 (LYG012560), two-component response regulator-like APRR2 (APRR2, LYG012584), phosphomevalonate kinase (PMVK, LYG012595), ARF11 (LYG014851), and NCED6 (LYG034219) (Table 2).

To validate the differential expression of candidate genes significantly associated with flower color-related phenotypes in $O$. fragrans, we performed RNA-seq analysis by sequencing nine transcriptomes of OFL (roots, stems, leaves, and flowers for six different flowering stages, with three biological replicates per sample). The results showed that of these 35 genes, 12 were differentially expressed during flowering (Fig. 5).

\section{Variations in CCD4 gene loci}

It was reported that the orange/red color of Osmanthus varieties in the 'Dangui group' was due to the accumulation of carotenoids ${ }^{10}$. We then analyzed the expression pattern of the $C C D$ gene family in different tissue parts (root, stem, and leaf) and flowering stages (S1-S6) of OFL. There were a total of four CCD4 genes in the OFL genome, including CCD4a (LYG004804) located on chromosome 2, CCD4b (LYG008494) and CCD4c (LYG008495) located on chromosome 4, and CCD4d (LYG026704) located on chromosome 15. The results showed that the $C C D 4 b, C C D 4 c$, and $C C D 4 d$ genes were differentially expressed during the flowering process and that the $C C D 4 a$ gene was expressed at a high level only in the root (Fig. 6).

Real-time PCR analysis was then carried out to screen for $C C D 4$ members that may contribute to orange/red petal color. The results showed that $C C D 4 b$ and $C C D 4 c$, located on chromosome 4, had abnormal gene structures and thus could not be cloned. The only functional member of $C C D 4$ that was differentially expressed during flowering was $C C D 4 d$, which was the same one identified in previous studies (Fig. 7) $8,9,12$.

Surprisingly, we found an allele with a 34-bp deletion in the first coding region of the CCD4d gene. The 34-bp deletion allele, denoted by 'a' (the wild allele is denoted by 'A'), existed in all varieties of the 'Dangui group'. We analyzed 122 resequenced samples, and the results showed that none of the genotype AA samples were from varieties in the 'Dangui group', that all genotype aa samples were varieties in the 'Dangui group', and that genotype Aa samples included varieties from all four groups, including those with white, yellow, and orange/red flower colors (Fig. 8, Supplementary Table 3). More interestingly, these Aa genotype samples in other groups were clustered closely to the 'Dangui group' on the phylogenetic tree (Fig. 3a). These results showed that the frameshift mutation of the $C C D 4$ gene is probably related to the formation of orange/red flower color in O. fragrans.

\section{Discussion}

As most cultivars of $O$. fragrans bloom in autumn, we chose two cultivars of $O$. fragrans, 'Liuyejingui' in the Jingui group and O. fragrans 'Gecheng Dangui' in the Dangui group, as plant materials for the preliminary experiment. The results showed that $O$. fragrans 'Gecheng Dangui' had a greater heterozygosity, 1.35\%, than 'Liuyejingui' (unpublished observation). Here, we present a genome for OFL, which is a typical autumn-flowering cultivar used for economic harvest and compare it with the published OFR genome ${ }^{13}$. Compared with that of the published genome, the size of the 'Liuyejingui' genome is similar; however, our contig N50 is $\sim 2.36 \mathrm{Mb}$, which is much larger, and our assembled and annotated BUSCO 
a

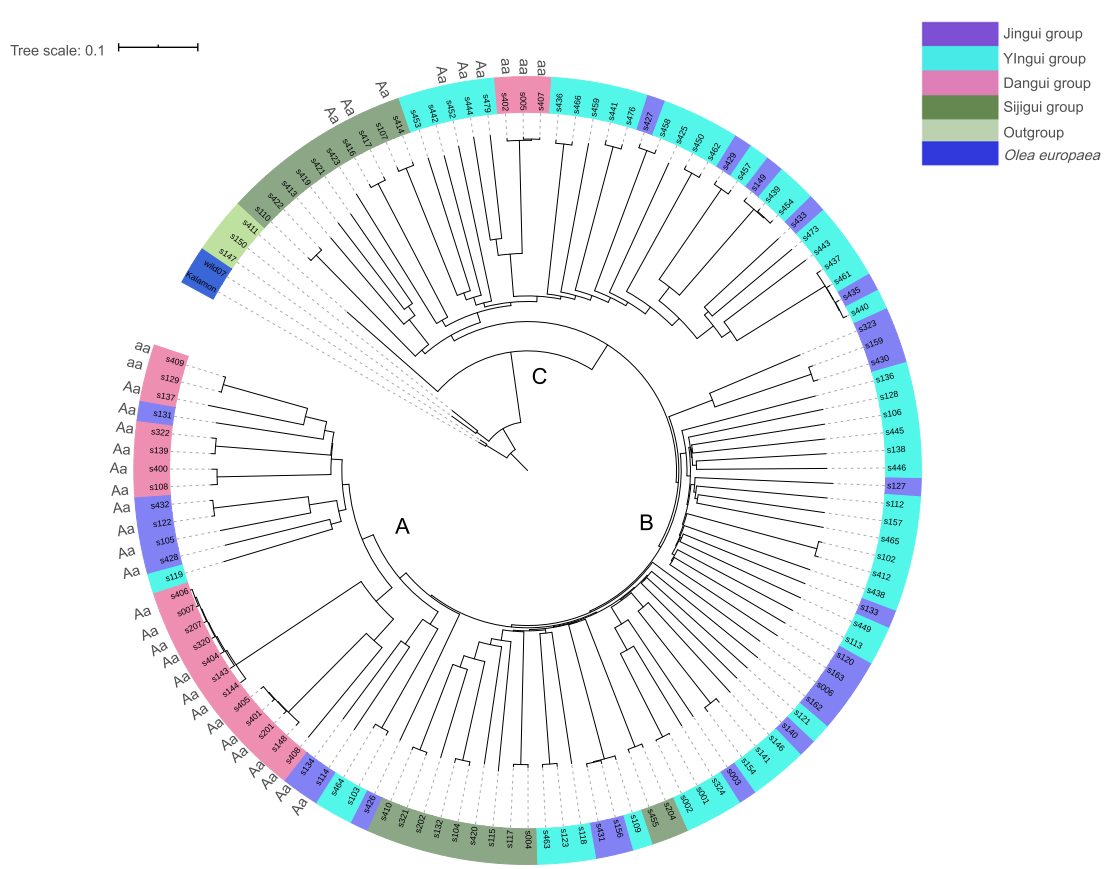

b
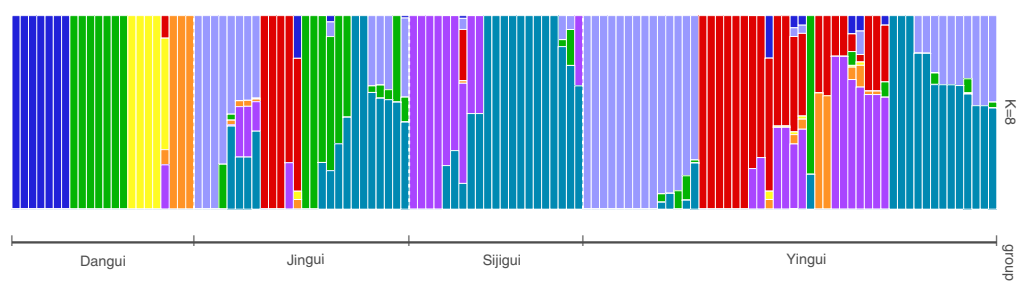

C

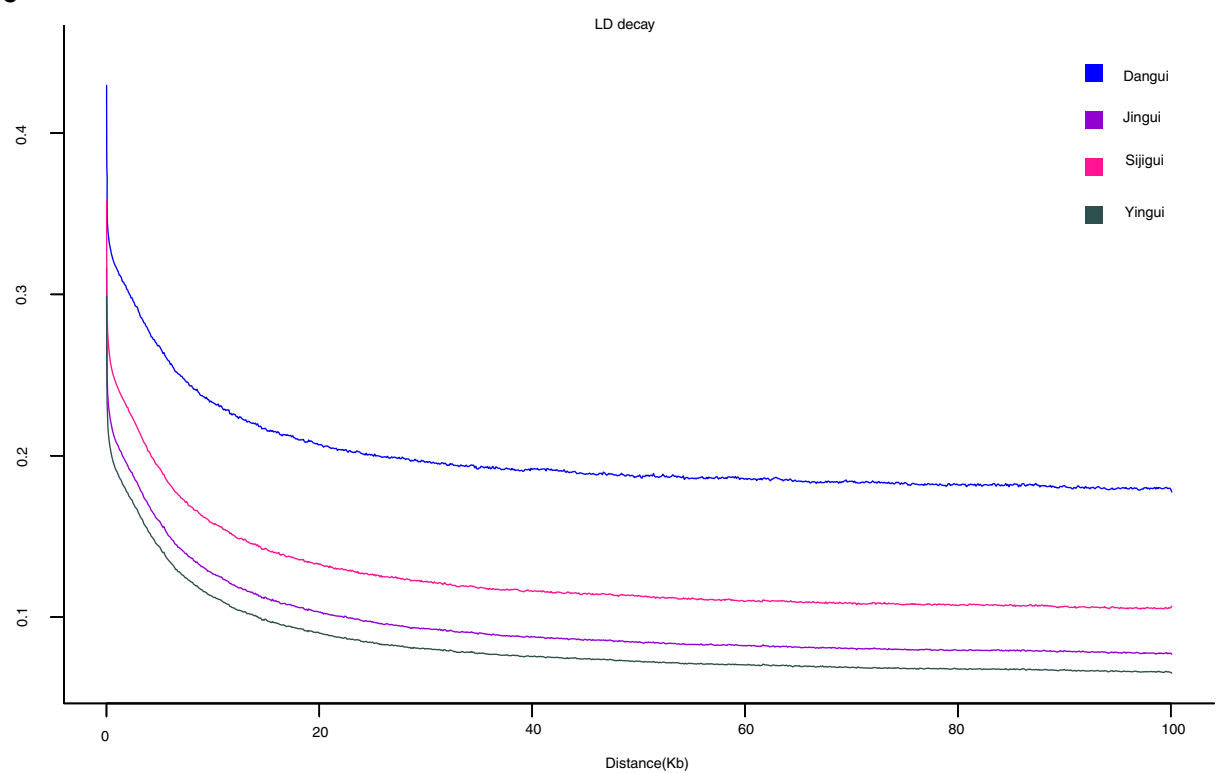

Fig. 3 Genomic variations and population evolution analysis. a Phylogenetic tree of 122 Osmanthus accessions and 2 Olea europaea; b population structure analysis; c LD plot 


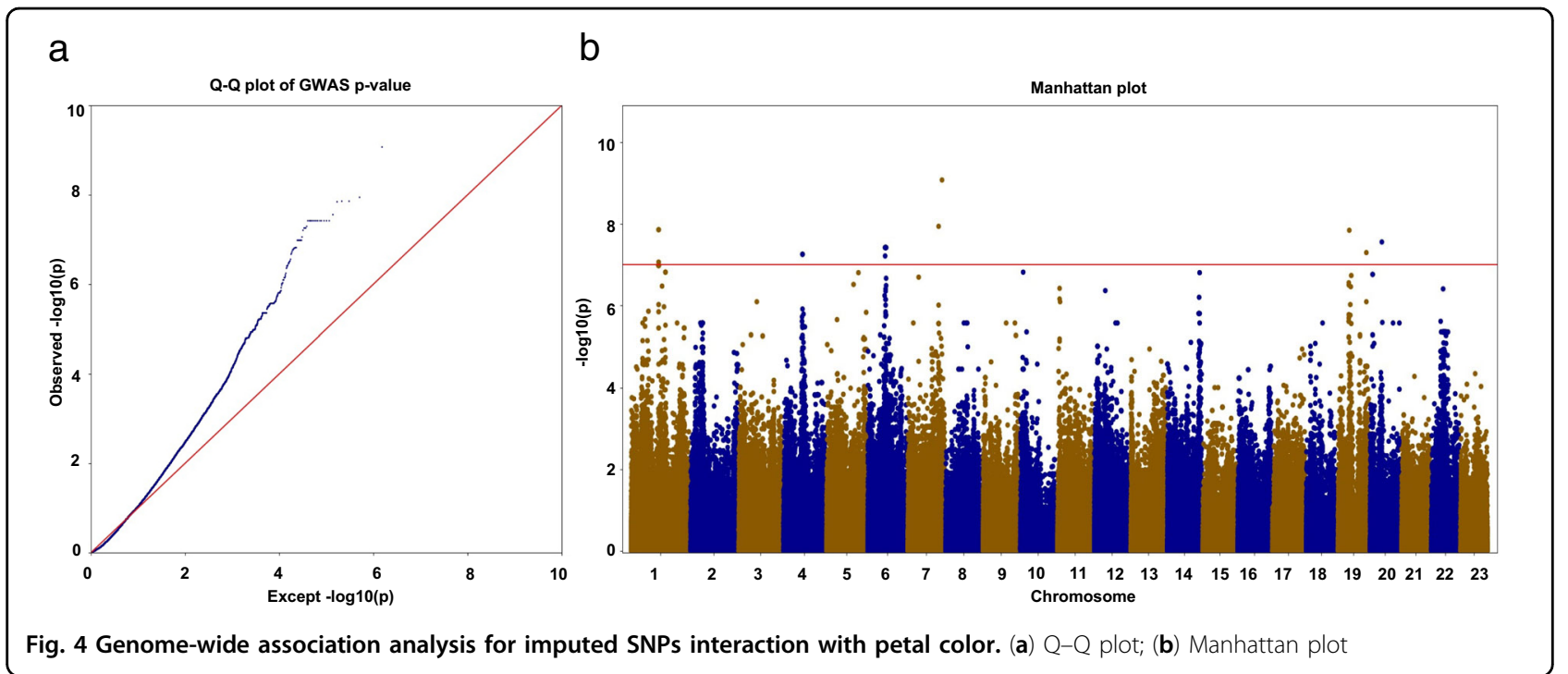

Table 2 SNPs associated with petal color

\begin{tabular}{|c|c|c|c|c|c|}
\hline Num. & Gene & SNP position & SNP $p$ value & FDR & Annotation \\
\hline 1 & LYG012558 & $6: 13817422$ & $3.74 \mathrm{E}^{-08}$ & 0.00146611 & RGG repeats nuclear RNA binding protein $A$ \\
\hline 2 & LYG012559 & $6: 13819023$ & $3.74 \mathrm{E}^{-08}$ & 0.00146611 & Phospholipase A1-Ibeta2, chloroplastic \\
\hline 3 & LYG012560 & & & & Ethylene-responsive transcription factor 2 \\
\hline 4 & LYG012563 & 6:13918308 & $6.10 \mathrm{E}^{-08}$ & 0.001891581 & uncharacterized \\
\hline 5 & LYG012568 & 6:14012303 & $3.74 \mathrm{E}^{-08}$ & 0.00146611 & Cyclin-dependent kinase D-1 \\
\hline 6 & LYG012579 & $6: 14272320$ & $3.74 \mathrm{E}^{-08}$ & 0.00146611 & Lon protease homolog 2, peroxisomal \\
\hline 7 & LYG012580 & & & & Protein phosphatase $2 \mathrm{C}$ \\
\hline 8 & LYG012581 & 6:14293904 & $3.74 \mathrm{E}^{-08}$ & 0.00146611 & Pentatricopeptide repeat-containing protein \\
\hline 9 & LYG012582 & & & & Type I inositol polyphosphate 5-phosphatase 2 \\
\hline 10 & LYG012584 & 6:14339148 & $3.74 \mathrm{E}^{-08}$ & 0.00146611 & Two-component response regulator-like APRR2 \\
\hline 11 & LYG012585 & $6: 14381541$ & $3.74 \mathrm{E}^{-08}$ & 0.00146611 & Haloacid dehalogenase-like hydrolase domain-containing protein \\
\hline 12 & LYG012586 & 6:14384154 & $3.74 \mathrm{E}^{-08}$ & 0.00146611 & Regulator of nonsense transcripts 1 \\
\hline 13 & LYG012587 & $6: 14392645$ & $3.74 \mathrm{E}^{-08}$ & 0.00146611 & AT-hook motif nuclear-localized protein 10 \\
\hline 14 & LYG012588 & 6:14413335 & $3.74 \mathrm{E}^{-08}$ & 0.00146611 & uncharacterized \\
\hline 15 & LYG012589 & & & & Protein CLMP1 \\
\hline 16 & LYG012595 & $6: 14571827$ & $3.74 \mathrm{E}^{-08}$ & 0.00146611 & Phosphomevalonate kinase, peroxisomal \\
\hline 17 & LYG012596 & & & & Extra-large guanine nucleotide-binding protein 3 \\
\hline 18 & LYG014851 & $7: 25078341$ & $1.13 \mathrm{E}^{-08}$ & 0.00146611 & Auxin response factor 11 \\
\hline 19 & LYG014852 & 7:27860295 & $8.32 \mathrm{E}^{-10}$ & 0.000618978 & uncharacterized \\
\hline 20 & LYG014853 & & & & L-ascorbate oxidase \\
\hline 21 & LYG033390 & 19:22751430 & $4.99 \mathrm{E}^{-08}$ & 0.001771043 & uncharacterized \\
\hline 22 & LYG033391 & & & & uncharacterized \\
\hline 23 & LYG033392 & & & & uncharacterized \\
\hline 24 & LYG034219 & 20:9512838 & $2.72 \mathrm{E}^{-08}$ & 0.00146611 & 9-cis-epoxycarotenoid dioxygenase NCED6, chloroplastic \\
\hline
\end{tabular}




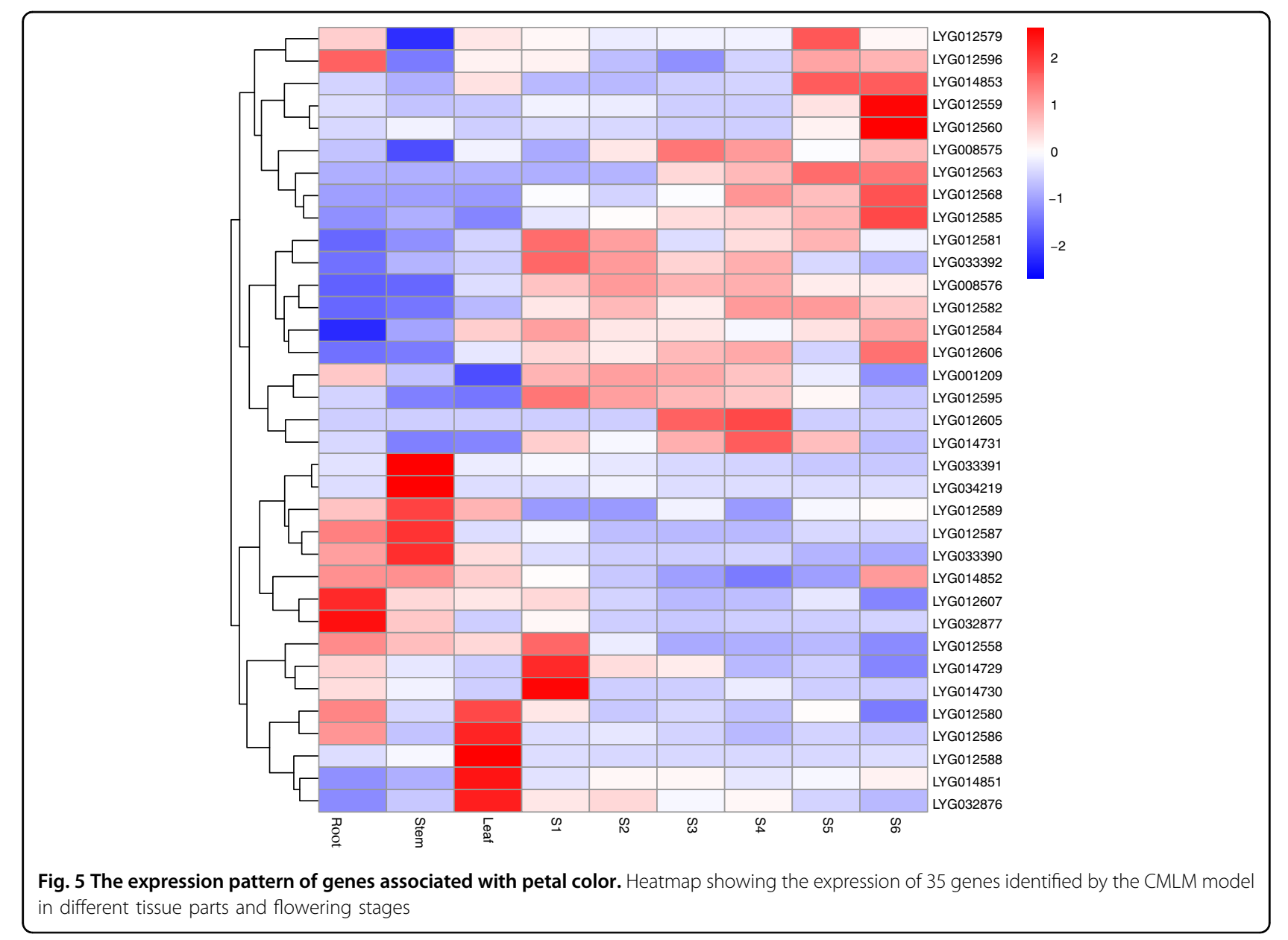

evaluation results are better (Table 1). The average length of our OFL genome gene is $\sim 5.6 \mathrm{~kb}$, which is higher than that in the published results, indicating that our gene structure annotation is more complete. Moreover, the calculated level of heterozygosity was $1.17 \%$ in $O$. fragrans 'Liuyejinggui', while it was higher (1.45\%) in OFR (Table 1). Synteny analyses were then performed, and the results showed a high collinearity between these two assemblies, except for some structural variation (Supplementary Fig. 3). In summary, we constructed a highquality reference genome.

As an important phenotypic trait, $O$. fragrans flower color has always been an essential basis for its classification and evolution ${ }^{66}$. Compared with the traditional morphological classification of the four groups of $O$. fragrans, the results of the population structure analysis show that the varieties are clustered into three groups. These results suggest that in the long-term evolutionary process, under the effects of natural and artificial selection, these varieties' genetic material has developed apparent regional clustering, which implies the different origins of $O$. fragrans in the southeastern and southwestern regions of China. ${ }^{1,2}$ This subpopulation differentiation caused by geographical isolation has also been reported in other species ${ }^{67-69}$.

It has been reported that the 'Sijigui group' is relatively close to the wild progenitor and that the 'Yingui group' is the more original of the three autumn-flowering groups; the 'Jingui group' appears later, and the 'Dangui group' appears latest $\mathrm{t}^{1,2,66}$. The white/yellowish-white flower color is likely the original character, according to the flower color analysis of wild species in Osmanthus. In contrast, the yellow to orange-red flower colors were not found in the wild species. They appear only under certain cultivation conditions, which indicates an evolutionary trait in the process of breed evolution. ${ }^{1}$ The results of LD decay analysis are consistent with the conclusions of traditional osmanthus resource surveys, with the exception of the 'Sijigui group', which suggests that varieties in the 'Sijigui group' are probably artificially domesticated species rather than wild species. DNA barcoding with the trnS-G and nad7 introns of 2 O. fragrans groups showed similar results: Sijigui and Dangui clustered together ${ }^{70}$.

In addition, compared with the other three groups of $O$. fragrans, most of the varieties in the 'Dangui group' eliminated their regional aggregation and clustered 


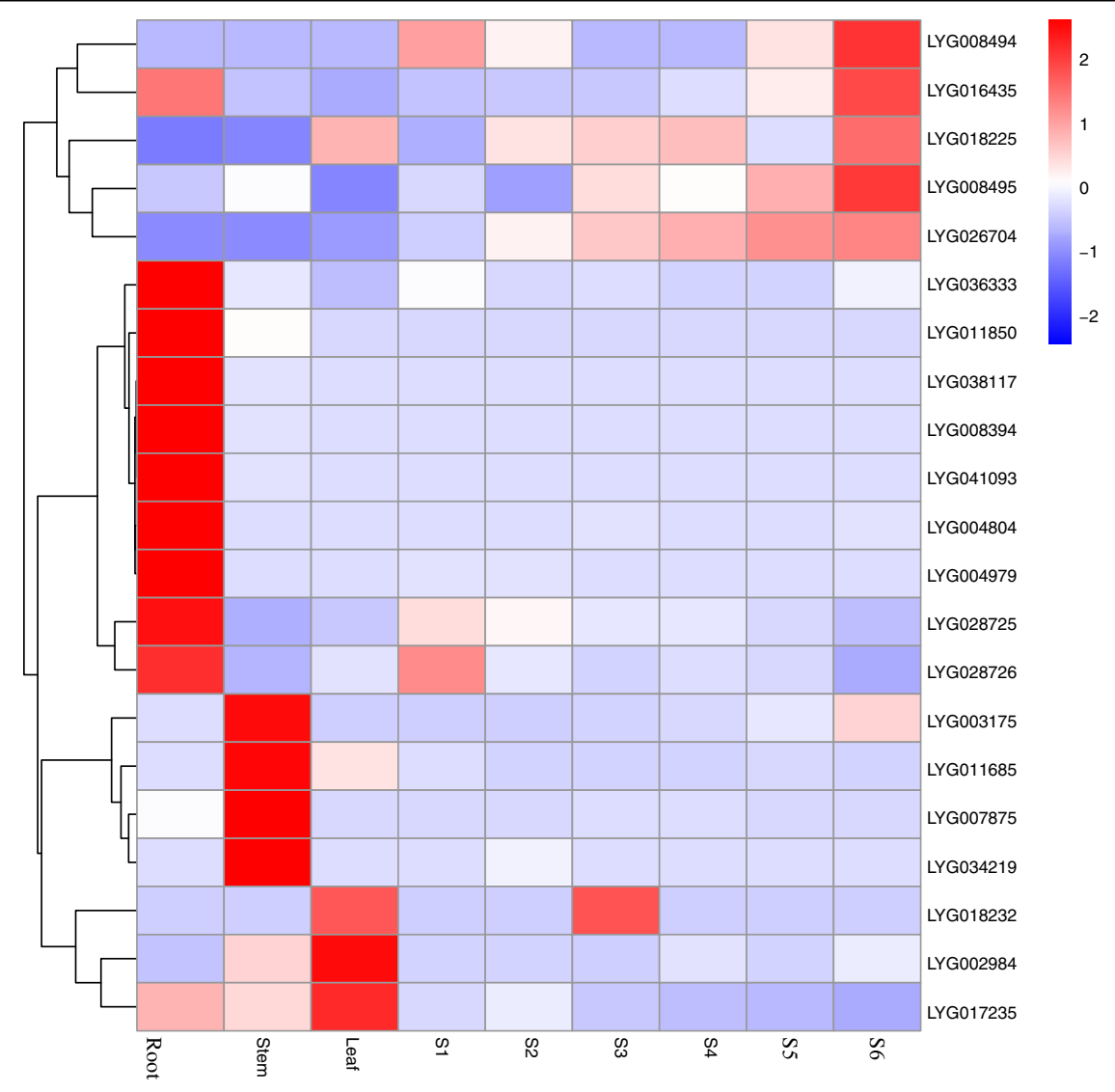

Fig. 6 The expression pattern of CCD gene family in $\mathbf{0}$. fragrans. Heatmap showing the expression of CCD genes in different tissue parts and flowering stages

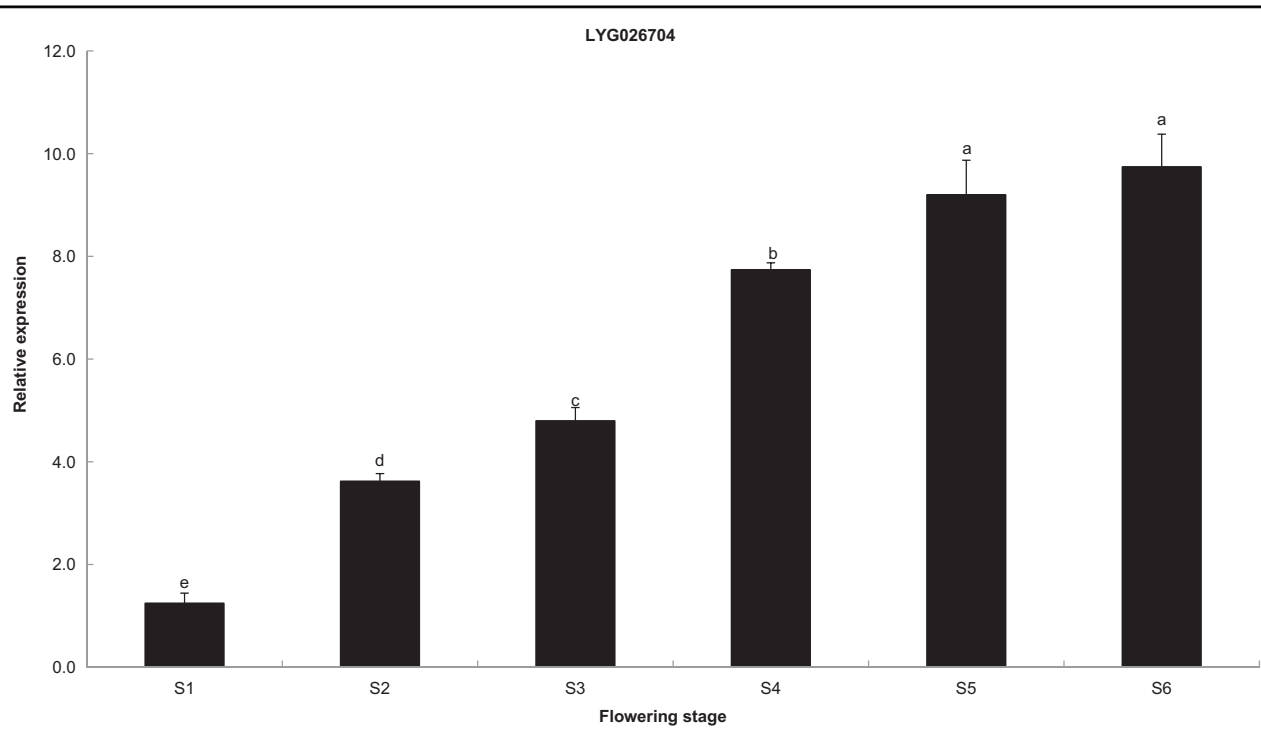

Fig. 7 Transcript levels of the $C C D 4 d$ gene in different flowering stages. Real-time PCR analysis of CCD $4 d$ genes in different flowering stages and the data represent the means \pm SD of the three replicates from three independent experiments with significant differences at $P=0.05$ level 


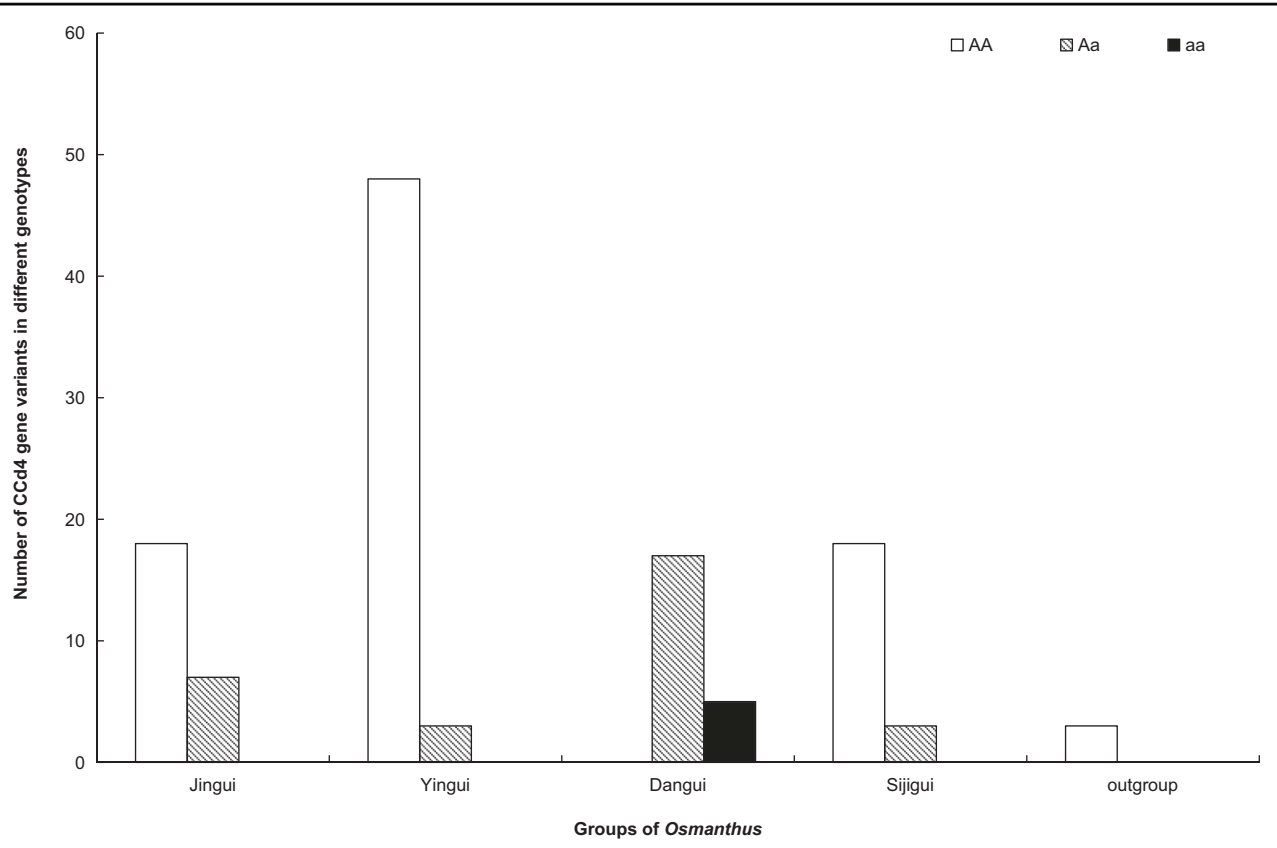

Fig. 8 The 34-bp deletion analysis of CCD4d gene. Numbers of CCD4d gene variants in different genotypes

independently, indicating that the 'Dangui group' was probably a bud sport that appeared in a particular area in the past. Under long-term artificial directional selection, a stable group of varieties was formed and then introduced and cultivated elsewhere. It has been suggested that the color of O. fragrans was described only as "white" in or before the Tang Dynasty in ancient Chinese texts but as both "white" and "yellow" during the Song Dynasty; the description of the red/orange color of $O$. fragrans appears only in the late Song Dynasty, which provides some support for our inference ${ }^{1,2}$.

To further explore the origin and evolution of flower color in O. fragrans, we identified significant QTLs and genomic regions associated with red/orange color through a GWAS in which several genes, such as PMVK, ERF2, and APRR2, were characterized. Among them, APRR2 has been reported to support carotenoid biofortification; it also increases the plastid number and area as well as pigment content, enhancing the levels of chlorophyll in immature unripe fruits and carotenoids in red ripe fruits when it is overexpressed ${ }^{71,72}$. ERF6 was reported to bind to the $C C D 4$ promoter and stimulate $C C D 4$ expression, thereby regulating the synthesis of $\beta$-ionone in O. fragrans petals9.

The differences in the flower color of $O$. fragrans varieties are attributable mainly to the level of carotenoids in the flowers ${ }^{10}$. Moreover, $C C D 1$ and $C C D 4$ are crucial contributors to the cleavage of $\alpha$-carotene and $\beta$-carotene ${ }^{11,12}$. The study of 'Redhaven' peach and its white-fleshed mutant showed that $C C D 1$ s contribute only to volatile production, while $C C D 4 \mathrm{~s}$ are likely to control carotenoid degradation ${ }^{73}$. In O. fragrans, the most crucial factor determining the diversity of carotenoid concentrations was also the differential expression level of $C C D 4^{9,10}$. In the present study, we found a surprising 34bp deletion in the first coding region of the CCD4d gene in all varieties of the "Dangui group", and this frameshift mutation existed in at least one site in both alleles. This result suggests that the orange/red color of the 'Dangui group' is probably related to the CCD4d mutation. Variations in CCD4 gene loci contribute to differences in carotenoid and apocarotenoid content among varieties of the same species and have also been found in citrus and petunia $^{74,75}$. On the other hand, the Aa genotype results occurring in the Jingui, Yingui, Dangui, and Sijigui phenotypes also indicated that the $C C D 4 d$ gene is probably not the only major gene that controls the biological metabolism of carotenoids. In addition to the CCD4d gene, there are likely other regulatory factors, such as ERF2 and APRR2, that were determined by GWAS to regulate the metabolism of carotenoids. Further studies should endeavor to study these candidate genes involved in flower color formation in order to elucidate the mechanism of the formation of orange/red color in $O$. fragrans flowers.

\section{Conclusion}

In this study, we successfully sequenced and assembled a reference genome for OFL, an autumn-flowering cultivar harvested for its economic value, by combining results 
from the Illumina, PacBio and $\mathrm{Hi}-\mathrm{C}$ platforms. We also reported on genomic variations and population evolution by resequencing 119 Osmanthus accessions from four groups of $O$. fragrans to explore the origin and evolution of flower color. Significant QTLs and genomic regions were identified in which several genes that were positively associated with petal color, such as ERF2 and APRR2, were located. On the other hand, the frameshift mutation of the CCD4 gene is probably related to the formation of orange/red flower color in $O$. fragrans. The reference genome sequence and genomic variation map of $O$. fragrans provide insights into the genome evolution of the $O$. fragrans species, benefiting both basic and applied plant biologists.

\section{Acknowledgements}

This work was supported by research grants provided by the National Natural Science Foundation of China (31600569 and 31700617), the Natural Science Foundation of Hubei Province (2017CFB235), the Nuclear Technology Innovation team project of Hubei University of Science and Technology (H2019002), and the Science and Technology Project of Xianning (2020NYYF02). We thank Shi Chunmei at the College of Horticulture \& Forestry Sciences of Huazhong Agricultural University and her colleagues for helping with the flow cytometry experiment.

\section{Author details}

'Hubei Engineering Research Center for Fragrant Plants, Hubei University of Science and Technology, Xianning 437100, China. ${ }^{2}$ Xianning Research Academy of Industrial Technology of Osmanthus fragrans, Xianning 437100, China. ${ }^{3}$ Key Laboratory of Horticultural Plant Biology, Ministry of Education, Huazhong Agricultural University, Wuhan 430070, China. ${ }^{4}$ Xianning Vocational Technical College, Xianning 437100, China. ${ }^{5}$ Xianning Forestry Academy of Sciences, Xianning 437100, China. ${ }^{6}$ Wuhan Frasergen Bioinformatics Co., Ltd., Wuhan 430070, China

\section{Author contributions}

H.C., J.Z., and C.W. designed and coordinated the whole project and the manuscript. J.Z., X.Z., X.C., and J.Y. led and carried out the whole project together. X.Y., S.X., and Y.S. performed the genome evolution analyses, gene family analyses, and metabolic analyses. J.Z., H.C., R.Z., and C.W participated in manuscript writing and revision. Q.F., Z.W., and J.L. collected the Osmanthus samples.

\section{Data availability}

Raw sequencing reads of all Osmanthus plant accessions reported in this study have been deposited into the public database of the National Center of Biotechnology Information (NCBI) BioProject under the accession number PRJNA679852. RNA-seq raw data were also deposited under these NCBI accessions.

\section{Conflict of interest}

The authors declare no competing interests.

Supplementary information The online version contains supplementary material available at https://doi.org/10.1038/s41438-021-00531-0.

Received: 5 December 2020 Revised: 8 February 2021 Accepted: 24 February 2021

Published online: 01 May 2021

\footnotetext{
References

1. Zang, D. K. \& Xiang, Q. B. Studies on Osmanthus fragrans cultivars. J. Nanjing Forestry Univ. 28, 7-13 (2004).
}

2. Xiang, Q. B. \& Liu, Y. L. An Illustrated Monograph of the Sweet Osmanthus Variety in China 93-260. (Zhejiang Science \& Technology Press, Hangzhou, Zhejiang Province, 2008).

3. He, Y. X., Yuan, W. J., Dong, M. F., Han, Y. J. \& Shang, F. D. The first genetic map in sweet osmanthus (Osmanthus fragrans Lour.) using specific locus amplified fragment sequencing. Front. Plant Sci. 8, 1621 (2017).

4. Duan, Y. F. et al. Genetic diversity of androdioecious Osmanthus fragrans (Oleaceae) cultivars using microsatellite markers. Appl. Plant Sci. 1, 1200092 (2013).

5. Cai, X. et al. Analysis of aroma-active compounds in three sweet osmanthus (Osmanthus fragrans) cultivars by gas-chromatogolfactometry and GC-mass spectrometry. J. Zhejiang. Univ. Sci. B. 15, 638-648 (2014).

6. $\mathrm{Fu}, \mathrm{J}$. X. et al. The Emission of the Floral Scent of Four Osmanthus fragrans Cultivars in Response to Different Temperatures. Molecules 22, 430 (2017).

7. $\mathrm{Fu}, \mathrm{J}$. X. et al. Identifcation of foral aromatic volatile compounds in 29 cultivars from four groups of Osmanthus fragrans by gas chromatography-mass spectrometry. Hortic. Environ. Biote. 60, 611-623 (2019).

8. Han, Y. J., Liu, L. X., Dong, M. F. \& Shang, F. D. cDNA cloning of the phytoene synthase (PSY) and expression analysis of PSY and carotenoid cleavage dioxygenase genes in Osmanthus fragrans. Biologia 68, 258-263 (2013).

9. Han, Y. J. et al. Mechanism of floral scent production in Osmanthus fragrans and the production and regulation of its key floral constituents, $\beta$-ionone and linalool. Hortic. Res. 6, 106 (2019).

10. Wang, Y. G. et al. Carotenoid accumulation and its contribution to flower coloration of Osmanthus fragrans. Front. Plant Sci. 9, 1499 (2018).

11. Baldermann, S. et al. Functional characterization of a carotenoid cleavage dioxygenase 1 and its relation to the carotenoid accumulation and volatile emission during the floral development of Osmanthus fragrans Lour. J. Exp. Bot. 61, 2967-2977 (2010).

12. Han, Y. J. et al. Characterization of OAWRKY3, a transcription factor that positively regulates the carotenoid cleavage dioxygenase gene OfCCD4 in Osmanthus fragrans. Plant Mol. Biol. 91, 485-496 (2016).

13. Yang, L. et al. The chromosome-level quality genome provides insights into the evolution of the biosynthesis genes for aroma compounds of Osmanthus fragrans. Hortic. Res. 5, 72 (2018).

14. Yang, K. M. Chinese Osmanthus 47-67 (China Forestry Publishing House, 2020).

15. Zou, J. J., Zhou, Y., Cai, X. \& Wang, C. Y. Increase in DNA fragmentation and the role of ethylene and reactive oxygen species in petal senescence of Osmanthus fragrans. Postharvest Biol. Tec. 93, 97-105 (2014).

16. Rogers, S. O. \& Bendich, A. J. Plant Molecular Biology Manual $73-83$ (eds Gelvin, S. B., Schilperoort, R. A. \& Verma, D. P. S.) (Springer, 1989).

17. Wang, B. et al. Unveiling the complexity of the maize transcriptome by singlemolecule long-read sequencing. Nat. Comms. 7, 11708 (2016).

18. Vaser, R., Sović, I., Nagarajan, N. \& Šikić, M. Fast and accurate de novo genome assembly from long uncorrected reads. Genome Res. 27, 737-746 (2017).

19. Walker, B. J. et al. Pilon: an integrated tool for comprehensive microbial variant detection and genome assembly improvement. Plos One 9, e112963 (2014).

20. Yang, X. et al. HTQC: a fast quality control toolkit for Illumina sequencing data. BMC Bioinforma. 14, 33 (2013).

21. Burton, J. N. et al. Chromosome-scale scaffolding of de novo genome assemblies based on chromatin interactions. Nat. Biotechnol. 31, 1119-1125 (2013).

22. Tarailo-Graovac, M. \& Chen, N. Using RepeatMasker to identify repetitive elements in genomic sequences. Curr. Protoc. Bioinformatics 4, 10.1-10.14 (2009).

23. Jurka, J. et al. Repbase update, a database of eukaryotic repetitive elements. Cytogenet. Genome Res. 110, 462-467 (2005).

24. Bao, Z. R. \& Eddy, S. R. Automated de novo identification of repeat sequence families in sequenced genomes. Genome Res. 12, 1269-1276 (2002).

25. Price, A. L., Jones, N. C. \& Pevzner, P. A. De novo identification of repeat families in large genomes. Bioinformatics 21, 1351-1358 (2005).

26. Xu, Z. \& Wang, H. LTR_FINDER: an efficient tool for the prediction of full-length LTR retrotransposons. Nucleic Acids Res. 35, W265-W268 (2007).

27. Benson, G. Tandem repeats finder: a program to analyze DNA sequences Nucleic Acids Res. 27, 573-580 (1999).

28. Stanke, M. et al. AUGUSTUS: ab initio prediction of alternative transcripts, Nucleic Acids Res. 34, W435-W439 (2006).

29. Burge, C. \& Karlin, S. Prediction of complete gene structures in human genomic DNA. J. Mol. Biol. 268, 78-94 (1997).

30. Slater, G. S. C. \& Birney, E. Automated generation of heuristics for biological sequence comparison. BMC Bioinforma. 6, 31 (2005). 
31. Birney, E. \& Durbin, R. Using GeneWise in the Drosophila annotation experiment. Genome Res. 10, 547-548 (2000).

32. $Y u, X$. J. et al. Detecting lineage-specific adaptive evolution of brain-expressed genes in human using rhesus macaque as outgroup. Genomics $\mathbf{8 8}, 745-751$ (2006).

33. Trapnell, C., Pachter, L. \& Salzberg, S. L. TopHat: discovering splice junctions with RNA-Seq. Bioinformatics 25, 1105-1111 (2009).

34. Trapnell, $C$. et al. Transcript assembly and quantification by RNA-Seq reveals unannotated transcripts and isoform switching during cell differentiation. Nat. Biotechnol. 28, 511-515 (2010).

35. Li, W. \& Godzik, A. Cd-hit: a fast program for clustering and comparing large sets of protein or nucleotide sequences. Bioinformatics 22, 1658-1659 (2006).

36. Wu, T. D. \& Colin, K. W. GMAP: a genomic mapping and alignment program for mRNA and EST sequences. Bioinformatics 21, 1859-1875 (2005).

37. Cantarel, B. L. et al. MAKER: An easy-to-use annotation pipeline designed for emerging model organism genomes. Genome Res. 18, 188-196 (2008).

38. Boeckmann, B. et al. The SWISS-PROT protein knowledgebase and its supplement TrEMBL in 2003. Nucleic Acids Res. 31, 365-370 (2003).

39. Mitchell, $A$. et al. The InterPro protein families database: the classification resource after 15 years. Nucleic Acids Res. 43, D213-D221 (2015).

40. Kanehisa, M. et al. KEGG for integration and interpretation of large-scale molecular data sets. Nucleic Acids Res. 40, D109-D114 (2012).

41. Altschul, S. F. et al. Gapped BLAST and PSI-BLAST: a new generation of protein database search programs. Nucleic Acids Res. 25, 3389-3402 (1997).

42. Camacho, C. et al. BLAST plus: architecture and applications. BMC Bioinform. 10, 421 (2009).

43. Mistry, J. et al. Predicting active site residue annotations in the Pfam database. BMC Bioinform. 8, 298 (2007)

44. Jones, P. et al. InterProScan 5: genome-scale protein function classification. Bioinformatics 30, 1236-1240 (2014)

45. Finn, R. D. et al. The Pfam protein families database. Nucleic Acids Res. $\mathbf{3 6}$ D281-D288 (2008).

46. Ashburner, M. et al. Gene Ontology: tool for the unification of biology. Nat. Genet. 25, 25-29 (2000).

47. Conesa, A. \& Gotz, S. Blast2GO: a comprehensive suite for functional analysis in plant genomics. Int. J. Plant Genom. 2008, 1-12 (2008).

48. Lowe, T. M. \& Eddy, S. R. tRNAscan-SE: a program for improved detection of transfer RNA genes in genomic sequence. Nucleic Acids Res. 25, 955-964 (1997).

49. Nawrocki, E. P., Kolbe, D. L. \& Eddy, S. R. Infernal 1.0: inference of RNA alignments. Bioinformatics 25, 1335-1337 (2009).

50. Li, L., Stoeckert, C. J. \& Roos, D. S. OrthoMCL: identification of ortholog groups for eukaryotic genomes. Genome Res. 13, 2178-2189 (2003).

51. Edgar, R. C. MUSCLE: multiple sequence alignment with high accuracy and high throughput. Nucleic Acids Res. 32, 1792-1797 (2004).

52. Stamatakis, A. RAxML version 8: a tool for phylogenetic analysis and postanalysis of large phylogenies. Bioinformatics 30, 1312-1313 (2014).

53. Han, M. V. et al. Estimating gene gain and loss rates in the presence of error in genome assembly and annotation using CAFE 3. Mol. Biol. Evol. 30, 1987-1997 (2013).

54. KieåBasa, S. M. et al. Adaptive seeds tame genomic sequence comparison. Genome Res. 21, 487-493 (2011).
55. Li, H. \& Durbin, R. Fast and accurate short read alignment with BurrowsWheeler transform. Bioinformatics 25, 1754-1760 (2009).

56. Poplin, R. et al. Scaling accurate genetic variant discovery to tens of thousands of samples. bioRxiv. 24, 1-14 (2018).

57. McKenna, A. et al. The genome analysis toolkit: a MapReduce framework for analyzing next-generation DNA sequencing data. Genome Res. 20, 1297-1303 (2010).

58. Letunic, I. \& Bork, P. Interactive Tree Of Life (iTOL) v4: recent updates and new developments. Nucleic Acids Res. 47, W256-W259 (2019).

59. Yang, J., Lee, S. H., Goddard, M. E. \& Visscher, P. M. GCTA: a tool for genomewide complex trait analysis. Am. J. Hum. Genet. 88, 76-82 (2011).

60. Alexander, D. H. et al. Fast model-based estimation of ancestry in unrelated individuals. Genome Res. 19, 1655-1664 (2009).

61. Zhang, C. et al. PopLDdecay: a fast and effective tool for linkage disequilibrium decay analysis based on variant call format files. Bioinformatics 10, 1093 (2018).

62. Danecek, P. et al. The variant call format and VCFtools. Bioinformatics 27, 2156-2158 (2011)

63. Tang, Y. et al. GAPIT version 2: an enhanced integrated tool for genomic association and prediction. Plant Genome 9, 1-9 (2016).

64. Benjamini, Y. \& Hochberg, Y. Controlling the false discovery rate: a practical and powerful approach to $\mathrm{m}$ utliple testing. J. R. Statis. Soc. B. $\mathbf{5 7}, \mathbf{2 8 9 - 3 0 0}$ (1995).

65. Dolezel, J., Greilhuber, J. \& Suda, J. Estimation of nuclear DNA content in plants using flow cytometry. Nat. Protoc. 2, 2233-2244 (2007).

66. Duan, Y. F. et al. Analysis on the current reseach situation of ancient sweet osmanthus and discussion on the origin and evolution of the sweet osmanthus cultivars in China. J. Hubei Univ. Natl 28, 375-379 (2010). 4.

67. Cun, Y. Z. \& Wang, X. Q. Plant recolonization in the Himalaya from the southeastern Qinghai-Tibetan Plateau: Geographical isolation contributed to high population differentiation. Mol. Phylogenet. Evol. 56, 972-982 (2010).

68. Gong, W. et al. Low genetic diversity and high genetic divergence caused by inbreeding and geographical isolation in the populations of endangered species Loropetalum subcordatum (Hamamelidaceae) endemic to China. Conserv. Genet. 11, 2281-2288 (2010).

69. Chen, J. H. et al. Genome-wide analysis of Cushion willow provides insights into alpine plant divergence in a biodiversity hotspot. Nat. commun. 10, 5230 (2019).

70. Wang, X. F. et al. Identification and RElationships of Osmanthus fragrans cultivar groups. J. Northeast Forestry Univ. 41, 71-74 (2013).

71. Pan, Y. et al. Network inference analysis identifies an APRR2-like gene linked to pigment accumulation in tomato and pepper fruits. Plant Physiol. 161, 1476-1485 (2013)

72. Oren, E. et al. The multi-allelic APRR2 gene is associated with fruit pigment accumulation in melon and watermelon. J. Exp. Bot. 70, 15 (2019).

73. Brandi, F. et al. Study of'Redhaven' peach and its white-fleshed mutant suggests a key role of CCD4 carotenoid dioxygenase in carotenoid and norisoprenoid volatile metabolism. BMC Plant Biol. 11, 24 (2011).

74. Zheng, X. J. et al. Natural variation in CCD4 promoter underpins speciesspecifific evolution of red coloration in citrus peel. Mol. Plant 12, 1294-1307 (2019).

75. Bodin, P. et al. Expression of CCD4 gene involved in carotenoid degradation in yellow-flowered Petunia $\times$ hybrida. Sci. Hortic. 261, 108916 (2020). 